\title{
Quantized Contact Transformations and Pseudodifferential Operators of Infinite Order
}

\author{
Dedicated to Professor Tosihusa Kimura on his 60th birthday
}

By

Takashi AokI*

\section{Introduction}

In this article we establish a formula which gives quantized contact transformations of pseudodifferential operators in terms of symbols. As an application of the formula, we define the characteristic sets by using symbols for pseudodifferential operators of infinite order and show that the sets are invariant under quantized contact transformations.

The notion of quantization for contact transformations was introduced by Sato, Kawai and Kashiwara [14]. They had been inspired by Egorov [6], Hörmander [7] and Maslov [13]. Roughly speaking, a quantization of a given contact transformation $\phi$ is an extension of $\phi$ to a ring isomorphism $\phi_{*}$ between the rings of pseudodifferential operators. We denote by $\Lambda$ the Lagrangean submanifold associated with $\phi$. Let $s$ be a non-degenerate section of a simple holonomic system with support $\Lambda$. Then the relation

$$
P\left(x, D_{x}\right) s=s Q\left(x^{\prime}, D_{x^{\prime}}\right) \quad\left(=Q^{*}\left(x^{\prime}, D_{x^{\prime}}\right) s\right)
$$

between two operators $P, Q$ gives a quantization $\phi_{*}$ by setting $Q$ $=\phi_{*}(P)$. Such a $\phi_{*}$ was first constructed in [14] for the sheaves $\mathscr{E}$ and $\mathscr{E} \infty$ of microdifferential operators of finite order and of infinite order, respectively. (In [14], the letter $\mathscr{P}$ was used instead of $\mathscr{E}$.) Kashiwara and Schapira [9] have established $\phi_{*}$ for the sheaf $\mathscr{E}^{\boldsymbol{R}}$ of pseudodifferential operators in a more general setting. Our first purpose is to write down the symbol of $Q=\phi_{*}(P)$ in terms of the symbol of $P$.

Quantized contact transformations play an important role in the study of partial differential equations. The importance is based on the invariance of the characteristic varieties under quantized contact transformations. For a

Communicated by T. Kawai, June 30, 1989. Revised October 16, 1989.

* Department of Mathematics and Physics, Kinki University, Osaka 577, Japan. 
microdifferential operator $P$ of finite order the characteristic variety $\mathrm{Ch}(P)$ of $P$ is defined to be the set of zero points of the principal symbol of $P$. We know the following two important properties of $\mathrm{Ch}(P)$ after [14]:

(i) The operator $P$ is invertible (as a microdifferential operator) outside $\mathrm{Ch}(P)$.

(ii) If $\phi_{*}$ is a quantization of a contact transformation $\phi$, we have

$$
\mathrm{Ch}\left(\phi_{*}(P)\right)=\phi(\mathrm{Ch}(P)) .
$$

The second subject of this paper is to extend the definition of $\operatorname{Ch}(P)$ for pseudodifferential operators $P$ of infinite order (i.e., $P \in \mathscr{E}^{\mathbb{R}}$ ) so that (i) and (ii) hold. For such operators the principal symbols do not make sense in general. By using "total" symbols, however, we can define $\mathrm{Ch}(P)$ in this case as follows. Let $P$ be an operator in $\mathscr{E}^{\mathbb{R}}$ and $P(x, \xi)$ the symbol (in the sense of Aoki [2]) of $P$. We say that a cotangent vector $x^{*}$ is non-characteristic with respect to $P$ if for each $\varepsilon>0$ there exists $C_{\varepsilon}>0$ such that

$$
|P(x, \xi)| \geq C_{\varepsilon} \exp (-\varepsilon|\xi|)
$$

holds in a conic neighborhood of $x^{*}$ at infinity. We write $\operatorname{Ch}(P)$ the complement of the set of all non-characteristic elements. Then (i) is a result of [3]. Moreover, we can see (ii) by using the symbol expression of $\phi_{*}(P)$. There is another way of defining characteristic sets by using the notion of microsupports [9]. Their definition has many good invariant properties. It is not easy, however, to determine their characteristic set for an arbitrary operator in $\mathscr{E}^{\boldsymbol{R}}$. In many interesting cases, we can write down $\mathrm{Ch}(P)$ exactly and, in general, we see that $\mathrm{Ch}(P)$ contains the characteristic set of $P$ in the sense of [9].

\section{\$1. Quantized Contact Transformations in Terms of Symbols}

1.1. Let $X$ be an open set in $C^{n}$. We denote by $T^{*} X$ the cotangent vector bundle of $X$. Let $\omega=\sum_{j=1}^{n} \xi_{j} d x_{j}$ be the holomorphic canonical 1-form on $T^{*} X$. Here $(x, \xi)=\left(x_{1}, \ldots, x_{n}, \xi_{1}, \ldots, \xi_{n}\right)$ denote local coordinates on $T^{*} X$. Let $\phi$ be a holomorphic contact (= homogeneous symplectic) transformation from $U_{1}$ to $U_{2}$, where $U_{1}$ and $U_{2}$ are conic open sets in $T^{*} X$. If we set $\left(x^{\prime}, \xi^{\prime}\right)=\phi(x, \xi)$, we have $\omega=\sum_{j=1}^{n} \xi_{j}^{\prime} d x_{j}^{\prime}$ by definition. We denote by $\Lambda_{0}$ the graph of $\phi$, i.e.,

$$
\Lambda_{0}=\left\{\left(x, \xi, x^{\prime}, \xi^{\prime}\right) \in T^{*} X \times T^{*} X \mid(x, \xi) \in U_{1}, \phi(x, \xi)=\left(x^{\prime}, \xi^{\prime}\right)\right\} .
$$

Now we set

$$
\Lambda=\left\{\left(x, x^{\prime}, \xi, \xi^{\prime}\right) \in T^{*}(X \times X) \mid\left(x, \xi, x^{\prime},-\xi^{\prime}\right) \in \Lambda_{0}\right\} .
$$

Then $\Lambda$ is a Lagrangean submanifold in $T^{*}(X \times X)$ with the canonical 1-form 
$\omega_{X \times X}=\sum_{j=1}^{n} \xi_{j} d x_{j}+\sum_{j=1}^{n} \xi_{j}^{\prime} d x_{j}^{\prime}$. We denote respectively by $\varphi_{1}$ and $\varphi_{2}$ the first and the second projections $T^{*}(X \times X) \rightarrow T^{*} X$. We have $\varphi_{1}(\Lambda)=U_{1}$ and $\varphi_{2}(\Lambda)=U_{2}^{a}$, where $a$ denotes the anti-podal map and $U_{2}^{a}=a\left(U_{2}\right)$. Let $\mathscr{M}$ be a simple holonomic system with support $\Lambda$ and $s$ a non-degenerate section of $\mathscr{M}$. We set

$$
\mathscr{M}^{\mathbf{R}}=\mathscr{E}_{X \times X}^{R} \underset{\mathscr{E}_{X \times X}}{\bigotimes} \mathscr{M}
$$

(For the definition of $\mathscr{E}^{\boldsymbol{R}}$, see [2], [9].) Then $\mathscr{M}^{\boldsymbol{R}}$ has a structure of $\left(\left.\mathscr{E}_{X}^{\boldsymbol{R}}\right|_{U_{1}},\left.\mathscr{E}_{X}^{\boldsymbol{R}}\right|_{U_{2}}\right)$-bi-module by using the following identifications:

$$
\begin{gathered}
\left.\left.\mathscr{E}_{X}^{R}\right|_{U_{1}} \simeq \varphi_{1}^{-1} \mathscr{E}_{X}^{\boldsymbol{R}}\right|_{\Lambda}, \\
\left.\left.\left.\mathscr{E}_{X}^{\boldsymbol{R}}\right|_{U_{2}} \stackrel{*}{\simeq} \mathscr{E}_{X}^{\mathbb{R}}\right|_{U_{2}^{a}} \simeq \varphi_{2}^{-1} \mathscr{E}_{X}^{\mathbb{R}}\right|_{\Lambda},
\end{gathered}
$$

where $*$ denotes the anti-isomorphism obtained by taking formal adjoint.

Theorem 1.1. The relation $P s=s Q\left(=Q^{*} s\right)$ between two operators $P \in \mathscr{E}_{X, p_{1}^{*}}^{\mathbf{R}}$ and $Q \in \mathscr{E}_{X, p_{2}^{*}}^{\boldsymbol{R}}\left(p_{1}^{*} \in U_{1}, p_{2}^{*}=\phi\left(p_{1}^{*}\right) \in U_{2}\right)$ induces a ring sheaf isomorphism

$$
\phi_{*}:\left.\mathscr{E}_{X}^{\boldsymbol{R}}\right|_{U_{1}} \longrightarrow \phi^{-1}\left(\left.\mathscr{E}_{X}^{\boldsymbol{R}}\right|_{U_{2}}\right)
$$

by setting $\phi_{*}(P)=Q$. Here $Q^{*} \in \mathscr{E}_{X, a\left(p_{2}^{*}\right)}^{\boldsymbol{R}}$ denotes the formal adjoint operator of $Q$ (with respect to the volume element $d x_{1}^{\prime} d x_{2}^{\prime} \ldots d x_{n}^{\prime}$ ).

We call $\phi_{*}$ a quantization of $\phi$. This theorem is a special case of [9, Theorem 11.1.1]. In our case, the restrictions of $\phi_{*}$ to $\left.\mathscr{E}_{X}\right|_{U_{1}}$ and to $\left.\mathscr{E}_{X}^{\infty}\right|_{U_{1}}$ give the following isomorphisms respectively:

$$
\begin{aligned}
& \phi_{*}:\left.\mathscr{E}_{X}\right|_{U_{1}} \rightarrow \phi^{-1}\left(\left.\mathscr{E}_{X}\right|_{U_{2}}\right), \\
& \phi_{*}:\left.\mathscr{E}_{X}^{\infty}\right|_{U_{1}} \longrightarrow \phi^{-1}\left(\left.\mathscr{E}_{X}^{\infty}\right|_{U_{2}}\right) .
\end{aligned}
$$

These isomorphisms were constructed by [14] and called quantized contact transformations.

1.2. Now we assume that $\phi$ is very close to the identity. We will write down the symbol of $\phi_{*}(P)$ for a special choice of $\mathscr{M}$.

Under the assumption for $\phi$, there are a conic open set $U_{0}$ in the $\left(x, \xi^{\prime}\right)$ space and a holomorphic function $\Omega\left(x, \xi^{\prime}\right)$ homogeneous of order 1 with respect to $\xi^{\prime}$ such that $\partial_{\xi^{\prime}} \Omega\left(x, \xi^{\prime}\right)$ and $\partial_{x} \Omega\left(x, \xi^{\prime}\right) /\left|\xi^{\prime}\right|$ are very small in $U_{0}$ and

$$
\Lambda_{0}=\left\{\left(x, \xi, x^{\prime}, \xi^{\prime}\right) \mid\left(x, \xi^{\prime}\right) \in U_{0}, x^{\prime}=x+\partial_{\xi^{\prime}} \Omega\left(x, \xi^{\prime}\right), \xi=\xi^{\prime}+\partial_{x} \Omega\left(x, \xi^{\prime}\right)\right\} .
$$

We choose the following system as $\mathscr{M}$ :

$$
\mathscr{M}=\mathscr{E}_{X \times X} / \mathscr{I}
$$


with

$$
\begin{aligned}
\mathscr{I}=\sum_{j=1}^{n} \mathscr{E}_{X \times X}\left(D_{x_{j}}+D_{x_{j}^{\prime}}+\right. & \left.\partial_{x_{j}} \Omega\left(x, D_{x^{\prime}}\right)\right) \\
& +\sum_{j=1}^{n} \mathscr{E}_{X \times X}\left(x_{j}-x_{j}^{\prime}+\partial_{\xi_{j}^{\prime}} \Omega\left(x, D_{x^{\prime}}\right)\right) .
\end{aligned}
$$

Here $D_{x_{j}}=\partial / \partial x_{j}, D_{x_{j}^{\prime}}=\partial / \partial x_{j}^{\prime}(j=1, \ldots, n)$ and $\partial_{x_{j}} \Omega\left(x, D_{x^{\prime}}\right)$ and $\partial_{\xi_{j}^{\prime}} \Omega\left(x, D_{x^{\prime}}\right)$ $(j=1, \ldots, n)$ denote the microdifferential operator of order 1 and 0 with (total) symbols $\partial_{x_{j}} \Omega\left(x, \xi^{\prime}\right)$ and $\partial_{\xi_{j}^{\prime}} \Omega\left(x, \xi^{\prime}\right)$, respectively. Notice that we distinguish the notation $\partial_{x}, \partial_{\eta}, \ldots$, etc., from $D_{x}, D_{\eta}, \ldots$, etc. For example, we use this notation as $\left[D_{x_{j}}, f\right]=D_{x_{j}} f-f D_{x_{j}}=\partial_{x_{j}} f$ to express the relation

$$
\frac{\partial}{\partial x_{j}}(f g)-f \frac{\partial g}{\partial x_{j}}=\frac{\partial f}{\partial x_{j}} \cdot g
$$

for each $g$.

Let $s$ be the residue class of $1_{X \times X}$ in $\mathscr{M}$. Intuitively, we are treating

$$
s=\int e^{\zeta \cdot\left(x-x^{\prime}\right)+\Omega(x, \zeta)} d \zeta
$$

Theorem 1.2. The relation $P s=s Q \quad\left(P \in \mathscr{E}_{X, p_{1}^{*}}^{\mathbb{R}}, Q \in \mathscr{E}_{X, p_{2}^{*}}^{\mathbb{R}} ; p_{1}^{*} \in U_{1}, p_{2}^{*}=\right.$ $\left.\phi\left(p_{1}^{*}\right) \in U_{2}\right)$ is equivalent to the following relation of symbols:

$$
\begin{aligned}
e^{\partial_{y} \cdot \partial_{\eta}} P\left(x, \eta+\xi^{\prime}\right. & \left.+\theta\left(x, x+y, \xi^{\prime}\right)\right)\left.\right|_{y=\eta=0} \\
& =\left.e^{\partial_{w} \cdot \partial_{\lambda}} Q\left(x+w+\vartheta\left(x, \xi^{\prime}, \xi^{\prime}+\lambda\right), \xi^{\prime}\right)\right|_{w=\lambda=0} .
\end{aligned}
$$

Here $P(x, \xi), Q\left(x^{\prime}, \xi^{\prime}\right)$ denote symbols of $P, Q$, respectively and $\theta, \vartheta$ are defined by

$$
\Omega\left(x+y, \xi^{\prime}\right)-\Omega\left(x, \xi^{\prime}\right)=y \cdot \theta\left(x, x+y, \xi^{\prime}\right),
$$

and

$$
\Omega\left(x, \xi^{\prime}+\lambda\right)-\Omega\left(x, \xi^{\prime}\right)=\lambda \cdot \vartheta\left(x, \xi^{\prime}, \xi^{\prime}+\lambda\right) .
$$

See [2] for the definition of symbols for operators in $\mathscr{E}^{\boldsymbol{R}}$. Precisely speaking, the formal sum in the left-hand side of (1.2), for example, reads the formal symbol

$$
\left.e^{t \partial_{y} \cdot \partial_{\eta}} P\left(x, \eta+\xi^{\prime}+\theta\left(x, x+y, \xi^{\prime}\right)\right)\right|_{y=\eta=0}
$$

in the sense of [2] and the equality holds modulo null-formal symbols.

Now we set

$$
\begin{aligned}
& \Theta_{j}=D_{x_{j}}+D_{x_{j}^{\prime}}+\partial_{x_{j}} \Omega\left(x, D_{x^{\prime}}\right), \\
& \Xi_{j}=x_{j}-x_{j}^{\prime}+\partial_{\xi_{j}^{\prime}} \Omega\left(x, D_{x^{\prime}}\right)
\end{aligned}
$$


for $j=1, \ldots, n$. Of course we have

$$
\begin{gathered}
{\left[\Theta_{j}, \Theta_{k}\right]=0, \quad\left[\Theta_{j}, \Xi_{k}\right]=0,} \\
{\left[\Xi_{j}, \Xi_{k}\right]=0}
\end{gathered}
$$

for $j, k=1, \ldots, n$. Let $p^{*}=\left(x_{0}, x_{0}^{\prime}, \xi_{0}, \xi_{0}^{\prime}\right)$ be a point on $\Lambda$. We set $p_{1}^{*}$ $=\varphi_{1}\left(p^{*}\right), p_{2}^{*}=a \circ \varphi_{2}\left(p^{*}\right)$ and $p^{* a}=\left(x_{0}, x_{0}^{\prime}, \xi_{0},-\xi_{0}^{\prime}\right)$. We denote by $S_{X \times X, p^{*}}$ the algebra of symbols of $\mathscr{E}_{X \times X, p^{*}}^{R}$ Let $\bar{S}_{X \times X, p^{*}}$ be the quotient of $S_{X \times X, p^{*}}$ by the ideal of null symbols. There is a linear isomorphism $\varpi: \bar{S}_{X \times X, p^{*}} \rightarrow \mathscr{E}_{X \times X, p^{*}}^{R}$ so that $\varpi\left(x_{j}\right)=x_{j}, \varpi\left(x_{j}^{\prime}\right)=x_{j}^{\prime}, \varpi\left(\xi_{j}\right)=D_{x_{j}}, \varpi\left(\xi_{j}^{\prime}\right)=D_{x_{j}^{\prime}}(j=1, \ldots, n)$. We often identify $\mathscr{E}_{X \times X, p^{*}}^{\mathbb{R}}$ with $\bar{S}_{X \times X, p^{*}}$ through this isomorphism. Let $M$ be the space of all (equivalence classes of) symbols $F \in \bar{S}_{X \times X, p^{* a}}$ such that $\left[F, x_{j}\right]=0(j=1, \ldots, n)$ in $\mathscr{E}_{X \times X, p^{* a}}^{\boldsymbol{R}}$ (i.e., $\partial F / \partial \xi_{j}=0(j=1, \ldots, n)$ in $\left.\bar{S}_{X \times X, p^{* a}}\right)$. If we write $f=f\left(x, x^{\prime}, \zeta\right)$ for $f \in M, \check{f}=f\left(x, x^{\prime},-\xi^{\prime}\right)$ is an element of $\bar{S}_{X \times X, p^{* a}}$. We set $\tilde{\Omega}=\tilde{\Omega}\left(x, x^{\prime}, \zeta\right)$ $=\Omega(x, \zeta)+\zeta \cdot\left(x-x^{\prime}\right)$. The following lemma is due to Malgrange ([12], Proposition 4.3).

Lemma 1.3. Let $F=F\left(x, x^{\prime}, D_{x}, D_{x^{\prime}}\right)$ be a differential operator defined in a neighborhood of $\left(x_{0}, x_{0}^{\prime}\right)$ and $f=f\left(x, x^{\prime}, \zeta\right)$ an element in $M$. Then we have

$$
\begin{gathered}
e^{-\tilde{\Omega}} F\left(e^{\tilde{\Omega}} f\right)=e^{\partial_{y} \cdot \partial_{\eta}+\partial_{w} \cdot \partial_{\lambda}} F\left(x, x^{\prime}, \zeta+\eta+\theta(x, x+y, \zeta), \lambda-\zeta\right) \times \\
\left.f\left(x+y, x^{\prime}+w, \zeta\right)\right|_{\substack{y=\eta=0 \\
w=\lambda=0}}
\end{gathered}
$$

Hence $e^{-\tilde{\Omega}} F\left(e^{\tilde{\Omega}} f\right) \in M$.

Proof. We may write

$$
\begin{aligned}
& e^{-\tilde{\Omega}} F\left(e^{\tilde{\Omega}} f\right)=e^{-\tilde{\Omega}\left(x, x^{\prime}, \zeta\right)} e^{\partial_{y} \cdot \partial_{\eta}+\partial_{w} \cdot \partial_{\lambda}} F\left(x, x^{\prime}, \eta, \lambda\right) \times
\end{aligned}
$$

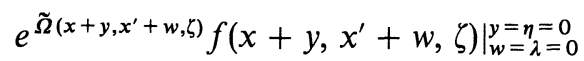

$$
\begin{aligned}
& =e^{\partial_{y} \cdot \partial_{\eta}+\partial_{w} \cdot \partial_{\lambda}} e^{y \cdot\left(\zeta+\theta\left(x, x^{\prime}+y, \zeta\right)\right)-w \cdot \zeta} F\left(x, x^{\prime}, \eta, \lambda\right) \times
\end{aligned}
$$

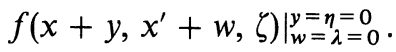

By using the following sublemma, we have (1.3).

Sublemma. Let $Q(y, \eta)$ be the (total) symbol of a differential operator $Q\left(y, D_{y}\right)$. Let $A(y, \eta)=\left(A_{1}(y, \eta), \ldots, A_{n}(y, \eta)\right)$ be an $n$-tuple of holomorphic functions. Then we have

$$
\begin{aligned}
\left.e^{\partial_{y} \cdot \partial_{\eta}}\left(e^{y \cdot A(y, \eta)} Q(y, \eta)\right)\right|_{y} & =0 \\
& =\left.e^{\partial_{y} \cdot \partial_{\eta}} \sum_{\alpha} \frac{1}{\alpha !} \partial_{\eta}^{\alpha}\left(A(y, \eta)^{\alpha} Q(y, \eta)\right)\right|_{y=0},
\end{aligned}
$$




$$
\begin{aligned}
\left.e^{\partial_{y} \cdot \partial_{\eta}}\left(e^{\eta \cdot A(y, \eta)} Q(y, \eta)\right)\right|_{\eta} & =0 \\
& =\left.e^{\partial_{y} \cdot \partial_{\eta}} \sum_{\alpha} \frac{1}{\alpha !} \partial_{y}^{\alpha}\left(A(y, \eta)^{\alpha} Q(y, \eta)\right)\right|_{\eta=0} .
\end{aligned}
$$

Proof of Sublemma. There are formal differential operators of infinite order $B_{j}=B_{j}\left(y, D_{\eta}, \eta\right)(j=1, \ldots, n)$ so that

$$
e^{D_{y} \cdot D_{\eta}}\left(e^{y \cdot A}-\sum_{\alpha} \frac{1}{\alpha !} D_{\eta}^{\alpha} A^{\alpha}\right)=e^{D_{y} \cdot D_{\eta}} \sum_{j=1}^{n}\left(y_{j}-D_{\eta_{j}}\right) B_{j}\left(y, D_{\eta}, \eta\right) .
$$

The right-hand side of this vanishes at $y=0$, for we know the following commutation relations:

$$
e^{D_{y} \cdot D_{\eta}}\left(y_{j}-D_{\eta_{j}}\right)=y_{j} e^{D_{y} \cdot D_{\eta}} \quad(j=1, \ldots, n) .
$$

Thus we have (1.4). A similar calculation shows (1.5).

Remark. (1.4) and (1.5) are valid not only for symbols of differential operators but also for general symbols so far as the right-hand sides make sense.

We denote by $L_{F}(f)$ the right-hand side of (1.3):

$$
\begin{aligned}
L_{F}(f)=e^{\partial_{y} \cdot \partial_{\eta}+\partial_{w} \cdot \partial_{\lambda}} F\left(x, x^{\prime}, \zeta+\eta+\theta(x, x+y, \zeta), \lambda-\zeta\right) \times & \\
& \left.f\left(x+y, x^{\prime}+w, \zeta\right)\right|_{\substack{y=\eta=0 \\
w=\lambda=0}} . \lambda
\end{aligned}
$$

Then it is easy to see $L_{F}(f)$ makes sense for every $F$ in $\mathscr{E}_{X \times X, p^{*}}^{R}$ as an element of $M$. Moreover, after some tedious calculation, we have

$$
L_{F_{1}}\left(L_{F_{2}}(f)\right)=L_{F_{1} F_{2}}(f)
$$

for every $F_{1}, F_{2} \in \mathscr{E}_{X \times X, p^{*}}^{\mathbb{R}}$. Thus we get a structure of $\mathscr{E}_{X \times X, p^{*}}^{\boldsymbol{R}}$-module on $M$. Let $N$ be the set of all elemetns of the form

$$
\sum_{j=1}^{n}\left(\frac{\partial a_{j}}{\partial \zeta_{j}}+\left(x_{j}-x_{j}^{\prime}+\partial_{\zeta_{j}} \Omega(x, \zeta)\right) a_{j}\right)
$$

for some $a_{j} \in M(j=1, \ldots, n)$.

Lemma 1.4. $N$ is an $\mathscr{E}_{X \times X, p^{*}}^{\mathbb{R}}$-submodule of $M$.

Proof. Let $f$ be an element in $N$ of the form (1.6). If we set $\check{a}_{j}=a_{j}\left(x, x^{\prime}\right.$, $\left.-\xi^{\prime}\right)$ then $\check{a}_{j}$ is a symbol in $\bar{S}_{X \times X, p^{*}}$. So we may regard $\check{a}_{j}$ as an operator in $\mathscr{E}_{X \times X, p^{*}}^{\boldsymbol{R}}$ Then we have

$$
L_{\Sigma_{j=1}^{n} \check{a}_{j} \Xi_{j}}(1)=f .
$$

Let $F$ be an operator in $\mathscr{E}_{X \times X, p^{*}}^{\mathbb{R}}$. Then 


$$
\begin{aligned}
L_{F}(f) & =L_{F}\left(L_{\Sigma_{J=1}^{n} \check{a}_{J} \Xi_{J}}(1)\right) \\
& =L_{\Sigma_{J=1}^{n}\left(F \check{a}_{J}\right) \Xi_{J}}(1) .
\end{aligned}
$$

By using the division theorem for $\mathscr{E}^{\boldsymbol{R}}[5$, Theorem 2.1], we write

$$
F \check{a}_{j}=\sum_{k=0}^{n} F_{j, k} \Theta_{k}+b_{j}
$$

in $\mathscr{E}_{X \times X, p^{*}}^{\boldsymbol{R}}$, where $b_{j}=b_{j}\left(x, x^{\prime}, D_{x^{\prime}}\right)$ commutes with $x_{k}$ for each $j, k$. Since $\left[\Theta_{k}, \Xi_{j}\right]=0$ and $L_{\Theta_{k}}(1)=0(j, k=1, \ldots, n)$, we have

$$
\begin{aligned}
L_{\Sigma_{j=1}^{n}\left(F \check{a}_{\jmath}\right) \Xi_{j}}(1) & =L_{\Sigma_{j, k}\left(F_{j, k} \Xi_{j}\right) \Theta_{k}}(1)+L_{\Sigma_{j} b_{\jmath} \Xi_{j}}(1) \\
& =\sum_{j}\left(\frac{\partial b_{j}^{\prime}}{\partial \zeta_{j}}+\left(x_{j}-x_{j}^{\prime}+\partial_{\zeta_{j}} \Omega(x, \zeta)\right) b_{j}^{\prime}\right),
\end{aligned}
$$

where $b_{j}^{\prime}=b_{j}\left(x, x^{\prime},-\zeta\right)$. Hence $L_{F}(f) \in N$.

Now we are interested in the quotient module $M / N$.

Lemma 1.5. The linear homomorphism

$$
L_{*}: \mathscr{E}_{X \times X, p^{*}}^{\boldsymbol{R}} F L_{F}(1) \in M
$$

induces an isomorphism $\mathscr{M}^{\mathbf{R}} \stackrel{\sim}{\longrightarrow} M / N$ as an $\mathscr{E}_{X \times X, p^{*}}^{\boldsymbol{R}}$-module.

Proof. Each $F \in \mathscr{E}_{X \times X, p^{*}}^{\boldsymbol{R}}$ can be uniquely written in the form

$$
F=\sum_{j=1}^{n} F_{j} \Theta_{j}+\bar{F}
$$

where $\bar{F}=\bar{F}\left(x, x^{\prime}, D_{x^{\prime}}\right)$ commutes with each $x_{j}(j=1, \ldots, n)$. Then $L_{F}(1)$ $=L_{\bar{F}}(1)=\bar{F}\left(x, x^{\prime},-\zeta\right)$. If $L_{F}(1)$ belongs to $N$, there are $F_{j}^{\prime}(j=1, \ldots, n)$ such that

$$
L_{F}(1)=L_{\Sigma_{j=1}^{n} F_{j}^{\prime} \Xi_{j}}(1)
$$

Hence there exist $F_{j}^{\prime \prime}(j=1, \ldots, n)$ so that

$$
F-\sum_{j} F_{j}^{\prime} \Xi_{j}=\sum_{j} F_{j}^{\prime \prime} \Theta_{j}
$$

Thus $F$ is in $\mathscr{I}$. Converse is trivial.

Proof of Theorem 1.2. By Lemma 1.5, the relation $P s=Q^{*} s$ is equivalent to $L_{P}(1) \equiv L_{Q^{*}}(1)(\bmod N)$. By definition, we have

$$
L_{P}(1)=\left.e^{\partial_{y} \cdot \partial_{\eta}} P(x, \zeta+\eta+\theta(x, x+y, \zeta))\right|_{y=\eta=0}
$$


and

$$
L_{Q^{*}}(1)=Q^{*}\left(x^{\prime},-\zeta\right)
$$

It remains to show

$$
Q^{*}\left(x^{\prime},-\zeta\right)=\left.e^{\partial_{w} \cdot \partial_{\lambda}} Q(x+w+\vartheta(x, \zeta, \zeta+\lambda), \zeta)\right|_{w=\lambda=0}
$$

in $M / N$. In this quotient space, we can write

$$
\begin{aligned}
\left(-\partial_{\zeta_{j}}\right)^{k} a & \equiv e^{-\left(\zeta \cdot\left(x-x^{\prime}\right)+\Omega(x, \zeta)\right)}\left(\partial_{\zeta_{j}}^{k} e^{\zeta \cdot\left(x-x^{\prime}\right)+\Omega(x, \zeta)}\right) a . \\
& \left.\equiv \partial_{\lambda_{j}}^{k} e^{\lambda \cdot\left(\left(x-x^{\prime}\right)+\vartheta(x, \zeta, \zeta+\lambda)\right)} a\right|_{\lambda_{j}=0}(\bmod N)
\end{aligned}
$$

for $j=1, \ldots, n ; k=0,1,2, \ldots$ Therefore, using (1.5), we have

$$
\begin{aligned}
Q^{*}\left(x^{\prime},-\zeta\right) & =\sum_{\alpha} \frac{(-1)^{|\alpha|}}{\alpha !} \partial_{x^{\prime}}^{\alpha} \partial_{\zeta}^{\alpha} Q\left(x^{\prime}, \zeta\right) \\
& =\left.e^{\partial_{w} \cdot \partial \lambda} e^{\lambda \cdot\left(\left(x-x^{\prime}\right)+\vartheta(x, \zeta, \zeta+\lambda)\right)} Q\left(x^{\prime}+w, \zeta\right)\right|_{w=\lambda=0} \\
& =\left.e^{\partial_{w} \cdot \partial \lambda} Q(x+w+\vartheta(x, \zeta, \zeta+\lambda), \zeta)\right|_{w=\lambda=0} .
\end{aligned}
$$

This completes the proof of Theorem 1.2.

Let $\mathscr{E}_{X \times X, p^{* a}}^{\boldsymbol{R}}$ be the set of all operators $A$ in $\mathscr{E}_{X \times X, p^{* a}}^{\boldsymbol{R}}$ which satisfy

$$
\left[x_{j}, A\right]=\left[D_{x_{j}^{\prime}}, A\right]=0, j=1, \ldots, n .
$$

By using the division theorem for $\mathscr{E}^{\boldsymbol{R}}, \mathscr{M}^{\boldsymbol{R}} \simeq M / N$ can be identified with $\mathscr{E}_{X \times X, p^{*}}^{\boldsymbol{R}}$. For $P \in \mathscr{E}_{X, p_{1}^{*}}^{\mathbb{R}}$ and $Q \in \mathscr{E}_{X, p_{2}^{*}}^{\mathbb{R}}$, we can regard $L_{P}(1)$ and $L_{Q^{*}}(1)$ as operators in ' $\mathscr{E}_{X \times X, p^{*}}^{\mathbb{R}}$.

Definition 1.6. We define two linear maps

$$
\begin{aligned}
& \Phi_{1}: \mathscr{E}_{X, p_{1}^{*}}^{\boldsymbol{R}} \longrightarrow{ }^{\prime} \mathscr{E}_{X \times X, p^{* a}}^{\boldsymbol{R}}, \\
& \Phi_{2}: \mathscr{E}_{X, p_{2}^{*}}^{\boldsymbol{R}} \longrightarrow{ }^{\prime} \mathscr{E}_{X \times X, p^{* a}}^{\boldsymbol{R}}
\end{aligned}
$$

by

$$
\begin{aligned}
& \Phi_{1}(P)=\left(L_{P}(1)\right)^{*} \\
& \Phi_{2}(Q)=\left(L_{Q^{*}}(1)\right)^{*}
\end{aligned}
$$

If we write $\Phi_{j}$ in terms of symbols we have two linear maps from $S_{X, p_{j}^{*}}$ to ' $S_{X \times X, p^{* a}}(j=1,2)$, where $S_{X, p_{j}^{*}}$ and ' $S_{X \times X, p^{* a}}$ denote the spaces of symbols of $\mathscr{E}_{X, p_{j}^{*}}^{\mathbb{R}}$ and ' $\mathscr{E}_{X \times X, p^{* a}}^{\mathbb{R}}$ respectively (cf. [2]). We use the same letters $\Phi_{j}$ for these maps. By the preceding arguments, we have

$$
\Phi_{1}(P)\left(x, \xi^{\prime}\right)=\left.e^{\partial_{y} \cdot \partial_{\eta}} P\left(x, \eta+\xi^{\prime}+\theta\left(x, x+y, \xi^{\prime}\right)\right)\right|_{y=\eta=0}
$$




$$
\Phi_{2}(Q)\left(x, \xi^{\prime}\right)=\left.e^{\partial_{w} \cdot \partial_{\lambda}} Q\left(x+w+\vartheta\left(x, \xi^{\prime}, \xi^{\prime}+\lambda\right), \xi^{\prime}\right)\right|_{w=\lambda=0} .
$$

By Theorems 1.1 and 1.2, the relation $\Phi_{1}(P)=\Phi_{2}(Q)$ gives $\phi_{*}$ by setting $\phi_{*}(P)$ $=Q$.

1.3. Heuristically $\Phi_{2}(Q)$ is obtained by calculating $e^{-\Omega}\left(e^{\Omega_{\circ}} Q\right)$, where $\circ$ means the composition of symbols by the Leibniz-Hörmander rule. So if we take the composition of the reversed symbol of $e^{-\Omega}$ and $e^{\Omega} \Phi_{2}(Q)$, we recover $Q$ up to an elliptic factor ([7], Kajitani and Wakabayashi [8], Kumano-go [11]). The following construction of $\Phi_{2}^{*}$ is based on this fact.

Let us consider the series

$$
B(z, \zeta, \eta)=\sum_{\beta} \frac{(-1)^{|\beta|}}{\beta !} \partial_{z}^{\beta}(\vartheta(z, \zeta, \eta))^{\beta} .
$$

Here $\vartheta$ was defined in Theorem 1.2. The series converges near $(z, \zeta, \eta)$ $=\left(x, \xi^{\prime}, \xi^{\prime}\right)$ for every $\left(x, \xi^{\prime}\right) \in U_{0}$, for $\vartheta$ is very small there, and defines a holomorphic function $B$ homogeneous of order 0 in $(\zeta, \eta)$. Let $\sigma=\sigma\left(x^{\prime}, \xi^{\prime}, \eta\right)$ be a holomorphic function satisfying

$$
\sigma\left(x^{\prime}, \xi^{\prime}, \eta\right)+\vartheta\left(x^{\prime}+\sigma\left(x^{\prime}, \xi^{\prime}, \eta\right), \xi^{\prime}, \eta\right)=0 .
$$

Such a $\sigma$ uniquely exists near $\left(x^{\prime}, \xi^{\prime}, \eta\right)=\left(x^{\prime}, \xi^{\prime}, \xi^{\prime}\right)$ for each $\left(x^{\prime}, \xi^{\prime}\right) \in U_{2}$ and it is homogeneous of order 0 in $\left(\xi^{\prime}, \eta\right)$.

Definition 1.7. For every symbol $F\left(x, \xi^{\prime}\right)$ in ' $S_{X \times X, p^{* a}}$, we set

$$
\begin{aligned}
& \Phi_{2}^{*}(F)\left(x^{\prime}, \xi^{\prime}\right) \\
& \quad=\left.e^{\partial_{y} \cdot \partial_{\eta}} B\left(x^{\prime}+y, \xi^{\prime}, \xi^{\prime}+\eta\right) F\left(x^{\prime}+y+\sigma\left(x^{\prime}+y, \xi^{\prime}, \xi^{\prime}+\eta\right), \xi^{\prime}\right)\right|_{y=\eta=0} .
\end{aligned}
$$

Clearly, $\Phi_{2}^{*}(F)$ is a symbol in $S_{X, p_{2}^{*}}$ and we have a linear map

$$
\Phi_{2}^{*}:{ }^{\prime} S_{X \times X, p^{* a}} \longrightarrow S_{X, p_{2}^{*}} .
$$

The following proposition shows that this map gives an "almost" inverse of $\Phi_{2}$.

Proposition 1.8. For every symbol $Q\left(x^{\prime}, \xi^{\prime}\right)$ in $S_{X, p_{2}^{*}}$, we have

$$
\Phi_{2}^{*}\left(\Phi_{2}(Q)\right)\left(x^{\prime}, \xi^{\prime}\right)=A \circ Q\left(x^{\prime}, \xi^{\prime}\right) .
$$

Here

$$
\begin{aligned}
A\left(x^{\prime}, \xi^{\prime}\right) & =\left.e^{\partial_{y} \cdot \partial_{\eta}} e^{\Omega\left(x^{\prime}+y, \xi^{\prime}\right)-\Omega\left(x^{\prime}+y, \xi^{\prime}+\eta\right)}\right|_{y=\eta=0} \\
& =\left.e^{\partial_{y} \cdot \partial_{\eta}} B\left(x^{\prime}+y, \xi^{\prime}, \xi^{\prime}+\eta\right)\right|_{y=\eta=0}
\end{aligned}
$$

is an elliptic microdifferential symbol of order 0 and $A \circ Q$ denotes the composite symbol of $A$ and $Q$ : 


$$
A \circ Q\left(x^{\prime}, \xi^{\prime}\right)=\left.e^{\partial_{w} \cdot \partial_{\lambda}} A\left(x^{\prime}, \xi^{\prime}+\lambda\right) Q\left(x^{\prime}+w, \xi^{\prime}\right)\right|_{w=\lambda=0} .
$$

Remark. By the hypothesis for $\phi$, we can assume that $A$ is defined near $p_{2}^{*}$.

To prove the proposition, we need the following lemma which gives the relation among $B, \sigma$ and $\vartheta$.

Lemma 1.9. Let $\psi(t), u_{j}(t)(j=1, \ldots, n)$ be holomorphic functions of $t$ $=\left(t_{1}, \ldots, t_{n}\right)$ defined in a neighborhood of $t=0$. Suppose that $u=\left(u_{1}, \ldots, u_{n}\right)$ is very small in $|t| \leq \varepsilon(\varepsilon>0)$ so that if $|t| \leq \varepsilon$ then $t+u(t)$ belongs to the neighborhood. Let $b(t)$ be a holomorphic function defined by

$$
b(t)=\sum_{\beta \geq 0} \frac{(-1)^{\beta}}{\beta !} \partial_{t}^{\beta} u(t)^{\beta} .
$$

Let $v(t)=\left(v_{1}(t), \ldots, v_{n}(t)\right)$ be an $n$-tuple of holomorphic functions determined by

$$
v(t)+u(t+v(t))=0 .
$$

Then we have

$$
\sum_{\alpha \geq 0} \frac{(-1)^{\alpha}}{\alpha !} \partial_{t}^{\alpha}\left(u(t)^{\alpha} \psi(t)\right)=b(t) \psi(t+v(t))
$$

for each sufficiently small $t$.

Proof. To prove (1.10), it suffices to show the following relation of formal differential operators of infinite order:

$$
\sum_{\alpha} \frac{(-1)^{\alpha}}{\alpha !} D_{t}^{\alpha} u(t)^{\alpha}=b(t) \sum_{\beta} \frac{v(t)^{\beta}}{\beta !} D_{t}^{\beta} .
$$

Let us consider a formal differential operator of infinite order

$$
T_{u}=\sum_{\alpha} \frac{u(t)^{\alpha}}{\alpha !} D_{t}^{\alpha}
$$

which assigns $\psi(t)$ to $\psi(t+u(t))$. It is invertible by the assumption and the inverse is the operator $\psi(t) \mapsto \psi(t+v(t))$, that is,

$$
T_{v}=\sum_{\beta} \frac{v(t)^{\beta}}{\beta !} D_{t}^{\beta},
$$

for we have $t+v(t)+u(t+v(t))=t$ and $t+u(t)+v(t+u(t))=t$ by the definition of $v$. This equals to the right-hand side of (1.11) up to $b$. The lefthand side of (1.11) is the formal adjoint $T_{u}^{*}$ of $T_{u}$. Let us compute the symbol of the composite operator $T_{u}^{*} T_{u}$. We denote by $\tau=\left(\tau_{1}, \ldots, \tau_{n}\right)$ the dual variables of $t$. The symbol of $T_{u}^{*}$ is 


$$
\left.e^{\partial_{t^{\prime}} \cdot \partial_{\tau^{\prime}}} e^{-u\left(t+t^{\prime}\right) \cdot\left(\tau+\tau^{\prime}\right)}\right|_{t^{\prime}=\tau^{\prime}=0} .
$$

Hence the symbol of $T_{u}^{*} T_{u}$ is

$$
\begin{aligned}
& e^{\partial_{t^{\prime \prime}} \cdot \partial_{\tau^{\prime \prime}}} e^{\partial_{t^{\prime}} \cdot \partial_{\tau^{\prime}}} e^{-u\left(t+t^{\prime}\right) \cdot\left(\tau+\tau^{\prime}+\tau^{\prime \prime}\right)} e^{u\left(t+t^{\prime \prime}\right) \cdot \tau}||_{t^{\prime \prime}=\tau^{\prime \prime}=0}^{t^{\prime}=0} \\
& =e^{\left(\partial_{t^{\prime}}+\partial_{t^{\prime \prime}}\right) \cdot \partial_{\tau^{\prime}}} e^{\left(u\left(t+t^{\prime \prime}\right)-u\left(t+t^{\prime}\right)\right) \cdot \tau-u\left(t+t^{\prime}\right) \cdot \tau^{\prime}}||_{t^{\prime \prime}=0}^{t^{\prime}=\tau^{\prime}=0} \\
& =\left.e^{\partial_{t^{\prime}} \cdot \partial_{\tau^{\prime}}} e^{-u\left(t+t^{\prime}\right) \cdot \tau^{\prime}}\right|_{t^{\prime}=\tau^{\prime}=0} \\
& =b(t) .
\end{aligned}
$$

This implies $T_{u}^{*} T_{u}$ is a multiplying operator by $b$. Thus we have

$$
\begin{aligned}
T_{u}^{*} & =\left(T_{u}^{*} T_{u}\right) T_{v} \\
& =b T_{v} .
\end{aligned}
$$

Proof of Proposition 1.8. By definition we have

$$
\begin{aligned}
& \Phi_{2}^{*}\left(\Phi_{2}(Q)\right)\left(x^{\prime}, \xi^{\prime}\right) \\
& \quad=\left.e^{\partial_{y} \cdot \partial_{\eta}+\partial_{w} \cdot \partial_{\lambda}} B\left(x^{\prime}+y, \xi^{\prime}, \xi^{\prime}+\eta\right) Q\left(x^{\prime}+y+w+Z, \xi^{\prime}\right)\right|_{w=\lambda=0} ^{y=\eta}=0
\end{aligned}
$$

with

$$
Z=\sigma\left(x^{\prime}+y, \xi^{\prime}, \xi^{\prime}+\eta\right)+\vartheta\left(x^{\prime}+y+\sigma\left(x^{\prime}+y, \xi^{\prime}, \xi^{\prime}+\eta\right), \xi^{\prime}, \xi^{\prime}+\lambda\right) .
$$

We set $y+w=z$ and $\eta-\lambda=\zeta$. Then we can rewrite (1.12) as

$$
\begin{aligned}
& \Phi_{2}^{*}\left(\Phi_{2}(Q)\right)\left(x^{\prime}, \xi^{\prime}\right) \\
& \quad=\left.e^{\partial_{y} \cdot \partial_{\zeta}+\partial_{z} \cdot \partial_{\lambda}} B\left(x^{\prime}+y, \xi^{\prime}, \xi^{\prime}+\zeta+\lambda\right) Q\left(x^{\prime}+z+Z^{\prime}, \xi^{\prime}\right)\right|_{\substack{y=\zeta=0 \\
z=\lambda}}=0
\end{aligned}
$$

with

$$
\begin{aligned}
Z^{\prime}= & \vartheta\left(x^{\prime}+y+\sigma\left(x^{\prime}+y, \xi^{\prime}, \xi^{\prime}+\zeta+\lambda\right), \xi^{\prime}, \xi^{\prime}+\lambda\right) \\
& -\vartheta\left(x^{\prime}+y+\sigma\left(x^{\prime}+y, \xi^{\prime}, \xi^{\prime}+\zeta+\lambda\right), \xi^{\prime}, \xi^{\prime}+\zeta+\lambda\right) .
\end{aligned}
$$

Here we have used the definition of $\sigma$. We put

$$
\begin{aligned}
& \sigma^{\prime}=\sigma\left(x^{\prime}+y, \xi^{\prime}, \xi^{\prime}+\zeta+\lambda\right), \\
& \vartheta_{1}=\vartheta\left(x^{\prime}+y, \xi^{\prime}, \xi^{\prime}+\lambda\right), \\
& \vartheta_{2}=\vartheta\left(x^{\prime}+y, \xi^{\prime}, \xi^{\prime}+\zeta+\lambda\right) .
\end{aligned}
$$

Then we may write

$$
Q\left(x^{\prime}+z+Z^{\prime}, \xi^{\prime}\right)=\sum_{\alpha} \frac{1}{\alpha !}\left(\sigma^{\prime}\right)^{\alpha} \partial_{y}^{\alpha}\left(e^{\left(\vartheta_{1}-\vartheta_{2}\right) \cdot \partial_{z}} Q\left(x^{\prime}+z, \xi^{\prime}\right)\right) .
$$

Applying Lemma 1.9 (or (1.11)) to the operator 


$$
B\left(x^{\prime}+y, \xi^{\prime}, \xi^{\prime}+\zeta+\lambda\right) \sum_{\alpha} \frac{1}{\alpha !}\left(\sigma^{\prime}\right)^{\alpha} D_{y}^{\alpha}
$$

and using the commutation relations

$$
\begin{aligned}
& {\left[e^{D_{y} \cdot D_{\zeta}}, \zeta_{j}\right]=e^{D_{y} \cdot D_{\zeta}} D_{y_{j}},} \\
& {\left[e^{D_{z} \cdot D_{\lambda}}, \lambda_{j}\right]=e^{D_{z} \cdot D_{\lambda}} D_{z_{J}},}
\end{aligned}
$$

we get

$$
\begin{aligned}
& \Phi_{2}^{*}\left(\Phi_{2}(Q)\right)\left(x^{\prime}, \xi^{\prime}\right) \\
& =\left.e^{\partial_{y} \cdot \partial_{\zeta}+\partial_{z} \cdot \partial_{\lambda}} \sum_{\alpha} \frac{(-1)^{\alpha}}{\alpha !} \partial_{y}^{\alpha}\left(\vartheta_{2}^{\alpha} e^{\left(\vartheta_{1}-\vartheta_{2}\right) \cdot \partial_{z}} Q\left(x^{\prime}+z, \xi^{\prime}\right)\right)\right|_{\substack{y=\zeta=0 \\
z=\lambda=0}} \\
& =e^{\partial_{y} \cdot \partial_{\zeta}+\partial_{z} \cdot \partial_{\lambda}} e^{-\zeta^{\prime} \cdot \vartheta_{2}+\lambda \cdot\left(\vartheta_{1}-\vartheta_{2}\right)} Q\left(x^{\prime}+z, \xi^{\prime}\right) \begin{array}{l}
y=\zeta=0 \\
z=\lambda=0 \\
\lambda=0
\end{array}
\end{aligned}
$$

Since

$$
-\zeta \cdot \vartheta_{2}+\lambda \cdot\left(\vartheta_{1}-\vartheta_{2}\right)=\Omega\left(x^{\prime}+y, \xi^{\prime}+\lambda\right)-\Omega\left(x^{\prime}+y, \xi^{\prime}+\zeta+\lambda\right),
$$

we conclude that

$$
\Phi_{2}^{*}\left(\Phi_{2}(Q)\right)\left(x^{\prime}, \xi^{\prime}\right)=\left.e^{\partial_{z} \cdot \partial_{\lambda}} A\left(x^{\prime}, \xi^{\prime}+\lambda\right) Q\left(x^{\prime}+z, \xi^{\prime}\right)\right|_{z=\lambda=0} .
$$

Corollary 1.10. For every symbol $F\left(x, \xi^{\prime}\right)$ in $S_{X \times X, p^{* a}}$, we have

$$
\Phi_{2}\left(A^{-1} \circ \Phi_{2}^{*}(F)\right)\left(x, \xi^{\prime}\right)=F\left(x, \xi^{\prime}\right) .
$$

Here $A^{-1}$ denotes the microdifferential symbol of order 0 such that $A^{-1} \circ A$ $=A \circ A^{-1}=1$.

Proof. It is sufficient to notice that $\Phi_{2}^{*}$ is injective. This can be proved by direct calculation of $\Phi_{2}\left(\Phi_{2}^{*}(F)\right)$ in the same way as in the proof of the proposition.

\subsection{Suppose that}

$$
\Phi_{1}(P)\left(x, \xi^{\prime}\right)=\Phi_{2}(Q)\left(x, \xi^{\prime}\right)
$$

for $P \in S_{X, p_{1}^{*}}$ and $Q \in S_{X, p_{2}^{*}}$. If we let $\Phi_{2}^{*}$ operate on the both members of (1.13), we have

$$
A \circ Q\left(x^{\prime}, \xi^{\prime}\right)=\Phi_{2}^{*}\left(\Phi_{1}(P)\right)\left(x^{\prime}, \xi^{\prime}\right)
$$

by Proposition 1.8. Conversely if we have (1.14), we recover (1.13) by Corollary 1.10. Thus we get

Theorem 1.11. Under the assumption of Section 1.2, the image $\phi_{*}(P)$ of $P \in \mathscr{E}_{X, p_{1}^{*}}^{\mathbb{R}}$ by the quantization $\phi_{*}$ of $\phi$ has a symbol $A^{-1} \circ \Phi_{2}^{*}\left(\Phi_{1}(P)\right)\left(x^{\prime}, \xi^{\prime}\right)$. 
If we write down the symbol $\Phi_{2}^{*}\left(\Phi_{1}(P)\right)\left(x^{\prime}, \xi^{\prime}\right)$ explicitly, we get

$$
\begin{aligned}
& \Phi_{2}^{*}\left(\Phi_{1}(P)\right)\left(x^{\prime}, \xi^{\prime}\right)=e^{\partial_{z} \cdot \partial_{\zeta}+\partial_{y} \cdot \partial_{\eta}} B\left(x^{\prime}+z, \xi^{\prime}, \xi^{\prime}+\zeta\right) \times \\
& \quad P\left(x^{\prime}+z+\sigma_{1}, \eta+\xi^{\prime}+\theta\left(x^{\prime}+z+\sigma_{1}, x^{\prime}+y+z+\sigma_{1}, \xi^{\prime}\right)\right) \substack{y=\eta=0 \\
z=\zeta=0}^{y=0}=0
\end{aligned}
$$

with $\sigma_{1}=\sigma\left(x^{\prime}+z, \xi^{\prime}, \xi^{\prime}+\zeta\right)$.

Remarks. (1) By definition, the principal part of $A\left(x^{\prime}, \xi^{\prime}\right)$ is equal to $B\left(x^{\prime}, \xi^{\prime}, \xi^{\prime}\right)$.

(2) Since $\theta\left(x, x, \xi^{\prime}\right)=\partial_{x} \Omega\left(x, \xi^{\prime}\right)$ and $\vartheta\left(x, \xi^{\prime}, \xi^{\prime}\right)=\partial_{\xi^{\prime}} \Omega\left(x, \xi^{\prime}\right)$, we have

$$
\phi^{-1}\left(x^{\prime}, \xi^{\prime}\right)=\left(x^{\prime}+\sigma_{0}\left(x^{\prime}, \xi^{\prime}\right), \xi^{\prime}+\partial_{x} \Omega\left(x^{\prime}+\sigma_{0}\left(x^{\prime}, \xi^{\prime}\right), \xi^{\prime}\right)\right),
$$

where we set $\sigma_{0}\left(x^{\prime}, \xi^{\prime}\right)=\sigma\left(x^{\prime}, \xi^{\prime}, \xi^{\prime}\right)$.

\section{§2. Characteristic Sets for Pseudodifferential Operators}

2.1. Let $U$ be a conic neighborhood of a point $x_{0}^{*}$ in $T^{*} X$. Let $P$ be a pseudodifferential operator defined in $U$, i.e., $P \in \mathscr{E}_{X}^{\boldsymbol{R}}(U)$. We fix a coordinate system $(x, \xi)$ in $T^{*} X$.

Definition 2.1. Let $x^{*}$ be an element of $U$ and $P(x, \xi)$ a symbol of $P$ defined in a conic neighborhood of $x_{0}^{*}$. The element $x^{*}$ is said to be noncharacteristic with respect to $P$ if for each $h>0$ there exists $C_{h}>0$ such that

$$
|P(x, \xi)| \geq C_{h} \exp (-h|\xi|)
$$

holds in $V \cap\{(x, \xi)|| \xi \mid \geq r\}$ for some conic neighborhood $V$ of $x^{*}$ and some constant $r>0$ which are independent of $h$. We denote by $\operatorname{Ch}(P)$ the complement in $T^{*} X$ of the set of all non-characteristic elements. We call $\mathrm{Ch}(P)$ the characteristic set of $P$.

Clearly, $\operatorname{Ch}(P)$ is a closed conic subset in $U$ and independent of the choice of the symbol $P(x, \xi)$. Note that, in general, $\mathrm{Ch}(P)$ is not invariant under the action of $C^{*}$ on $T^{*} X$. If $x^{*} \notin \mathrm{Ch}(P), 1 / P(x, \xi)$ is a symbol (i.e., of infraexponential type in $\xi$ ) near $x^{*}$. Hence it follows immediately from $[3$, Theorem 5.1] that

Theorem 2.2. If $x^{*} \notin \mathrm{Ch}(P)$ then $P$ is invertible in $\mathscr{E}_{X, x^{*}}^{\boldsymbol{R}}$.

If $1 / P(x, \xi)$ is a symbol, we may assume $P$ has a symbol of the form

$$
P(x, \xi)=e^{p(x, \xi)}
$$

near $x^{*}$. Here $p(x, \xi)$ is a symbol of order $1-0$ in the sense of [2]. For the characteristic set of the formal adjoint $P^{*}$ of $P$, we have 
Theorem 2.3. $\mathrm{Ch}\left(P^{*}\right)=a(\mathrm{Ch}(P))$, where a denotes the anti-podal map.

In fact, if $P$ has a symbol of the form (2.1), the formal adjoint $P^{*}$ also has an exponential symbol

$$
e^{p^{*}(x, \xi)}
$$

with some symbol $p^{*}(x, \xi)$ of order $1-0$ defined near $a\left(x^{*}\right)$ (see $[1$, Theorem 3.3]). Hence we have $a\left(x^{*}\right) \notin \mathrm{Ch}\left(P^{*}\right)$.

2.2. Let $\phi, U_{1}, U_{2}, \phi_{*}$ be as in Section 1. As we have remarked, if a point $x^{*}$ in $U$ does not belong to $\mathrm{Ch}(P)$, we may assume that $P$ has a symbol of the form (2.1) near $x^{*}$. Combining Theorem 1.11 and the discussion of [1, Sections 3 and 5], we see that

$$
\Phi_{2}^{*}\left(\Phi_{1}\left(e^{p}\right)\right)\left(x^{\prime}, \xi^{\prime}\right)
$$

can be written in the form

$$
e^{q\left(x^{\prime}, \xi^{\prime}\right)}
$$

with a symbol $q\left(x^{\prime}, \xi^{\prime}\right)$ of order $1-0$ defined near $\phi\left(x^{*}\right)$. (See the second remark after Theorem 1.11.) This implies $\phi\left(x^{*}\right) \notin \mathrm{Ch}\left(\phi_{*}(P)\right)$ (recall that $A$ is elliptic). Hence we have $\mathrm{Ch}\left(\phi_{*}(P)\right)=\phi(\mathrm{Ch}(P))$.

We know that we can never canonically specify the quantized contact transformation for a given contact transformation (cf. [14]). But it is determined up to inner automorphisms by elliptic microdifferential operators. Such ambiguity does not affect the definition of non-characteristic elements. Therefore if we choose another quantization $\phi_{*}^{\prime}$ of $\phi$, we have

$$
\operatorname{Ch}\left(\phi_{*}^{\prime}(P)\right)=\operatorname{Ch}\left(\phi_{*}(P)\right) .
$$

Moreover, any contact transformation can be obtained by successive application of such transformations as in Section 1.2. Thus we have

Theorem 2.4. Let $\phi$ be an arbitrary contact transformation and $\phi_{*} a$ quantization. Then we have $\mathrm{Ch}\left(\phi_{*}(\cdot)\right)=\phi(\mathrm{Ch}(\cdot))$.

In particular, $\mathrm{Ch}(\cdot)$ does not depend on the choice of the coordinate system in $X$. Of course, if $P$ is a microdifferential operator of finite order, $\mathrm{Ch}(P)$ coincides with the set of all zeros of the principal symbol of $P$. In this case $\mathrm{Ch}(P)$ is $C^{*}$-invariant. When $P$ is a differential operator of infinite order, our definition of $\operatorname{Ch}(P)$ is equivalent to that in [4]. Moreover if $P$ is with constant coefficients, our characteristic set of $P$ is the same as $V(P)$ in Kawai [10].

By Theorem 2.2, $\mathrm{Ch}(P)$ contains $\operatorname{supp}\left(\mathscr{E}_{X}^{\mathbb{R}} / \mathscr{E}_{X}^{R} P\right)$ which is called the characteristic variety of $\mathscr{E}_{X}^{R}$-module $\mathscr{E}_{X}^{R} / \mathscr{E}_{X}^{R} P$ in [9]. 


\section{References}

[1] Aoki, T., Calcul exponentiel des opérateurs microdifférentiels d'ordre infini, I, Ann. Inst. Fourier, Grenoble, 33-4 (1983), 227-250.

[2] — Symbols and formal symbols of pseudodifferential operators, Adv. Stud. Pure Math., 4 (1984), 181-208.

[3] - Calcul exponentiel des opérateurs microdifférentiels d'ordre infini, II, Ann. Inst. Fourier, Grenoble, 36-2 (1986), 143-165.

[4] - Existence and continuation of holomorphic solutions of differential equations of infinite order, $A d v$. in Math., 72 (1988), 261-283.

[5] Aoki, T., M. Kashiwara, and T. Kawai, On a class of linear differential operators of infinite order with finite index, Adv. in Math., 62 (1986), 155-168.

[6] Egorov, Yu. V., On canonical transformations of pseudodifferential operators, Uspehi Mat. Nauk, 24-5 (1969), 235-236.

[7] Hörmander, L., Fourier integral operators, I, Acta Math., 127 (1971), 79-183.

[8] Kajitani, K., and S. Wakabayashi, Microhyperbolic operators in Gevrey classes, Publ. RIMS, Kyoto Univ., 25 (1989), 169-221.

[9] Kashiwara, M., and P. Schapira, Microlocal Study of Sheaves, Astérisque 128, 1985.

[10] Kawai, T., On the theory of Fourier hyperfunctions and its applications to partial differential equations with constant coefficients, J. Fac. Sci., Univ. Tokyo, Sect. IA, 17 (1970), 467-517.

[11] Kumano-go, H., Pseudo-differential Operators, MIT Press, 1981.

[12] Malgrange, B., L'involutivité des caractétistiques des systèmes différentiels et microdifférentiels, Sém. Bourbaki 1977/78, no. 522.

[13] Maslov, V., Theory of Perturbation and Asymptotic Method, Moscow State Univ., 1965.

[14] Sato, M., T. Kawai, and M. Kashiwara, Microfunctions and pseudo-differential equations, Lecture Notes in Math., 287 (1973), 265-529. 
